\title{
Frequency and Time Domain responses of Cubic Quintic Septic Duffing Oscillator
}

\author{
S. K. Remmi ${ }^{a}$, M. M. Latha ${ }^{b}$
}

${ }^{a}$ Department of Physics, Women's Christian College, Nagercoil - 629 001, India

(Affiliated to Manonmaniam Sundaranar University, Abishekapatti, Tirunelveli- 627

012, Tamilnadu, India)

${ }^{\mathrm{b}}$ Associate Professor, Department of Physics, Women's Christian College , Nagercoil - 629

001, India

\section{Abstract}

In this paper, the harmonic response of a simple lumped parameter model of a forced nonlinear Duffing oscillator is characterized. The Duffing oscillator is modelled using 3 springs of prescribed material stiffness and critical geometrical parameter. The spring force and the forcing factor add more nonlinearity to the system which is governed by Cubic Quintic Septic odd power differential equation. The solutions to this strongly nonlinear system are determined analytically using Homotopy Analysis method (HAM). By this approximation method, the system response as the increase in amplitude of the periodic forcing is studied. In the frequency domain the amplitude response exhibits hysteresis and in the time domain the system undergoes stable and unstable limit cycles for various initial conditions.

\section{Introduction}

The Duffing oscillator associated with damping is a simple model that shows different types of oscillations such a chaos and limit cycles through several bifurcations. Nowadays many researchers are inspired to probe on such nonlinear differential equations associated with Duffing Oscillator, since its characteristics replicates similar dynamics in our day today life. Oscillations of nonlinear systems under the action of a periodic external force bears much more physical significance due to many interesting features behind it. It is not very simple to analyze such nonlinear problems because of nonlinear spring force caused by physical constraints in material and setup, linear viscous damping, and periodic forcing. But there are several approximation methods to explore the stability of periodic responses and their potential in highlighting the behaviour of other nonlinear phenomena. For many years, multiple scales and averaging methods [1] have been used to obtain approximate periodic solutions for weakly nonlinear systems. However, for some parameter values convergence cannot be achieved in such analysis. More recently, the Harmonic Balance method has been widely used to study approximately the convergence of stable and unstable periodic responses of nonlinear oscillators [2,3] for sufficiently large number of harmonics. But the algebraic manipulations lead to messy solutions which are difficult to identify except for the experienced analyst. On the other hand, the oscillations of nonlinear damped system have two different time scales related to frequency of oscillation and the decaying amplitude of oscillation. Thus, perturbation and asymptotic methods are often valid only for weakly nonlinear problems and found difficult to solve the strong nonlinear partial differentiation equations (PDEs) by means of analytic methods. However, In 1992, one such kind of analytic approximation method was proposed by Liao namely the HAM [4-8] to investigate strong nonlinear PDEs which enrich our physical understandings about the nonlinear phenomena.

Duffing oscillators are extremely nonlinear even in its unforced condition. Hence it is certain that in the externally excited state, such system exhibits a stunning behavior in their 
dynamic responses. Hopefully, no researches have done a detailed dynamics about a system described by a Cubic Quintic Septic Duffing (CQSD) equation with different time scales under HAM. This paper presents the dynamics of three springs carrying equal stiffness constructed geometrically with negative and positive stiffness elements which are kept at a particular oblique angle of Quasi Zero Stiffness condition subjected to periodic forcing. The paper is systemized as follows: Details of the model setup and the configuration of three-spring damping system for optimized QZS condition are given in Section 2. In section 3 we discuss about the HAM to arrive the frequency amplitude relation. Section 4 describes the frequency response curve of the three-spring system. The time domain of the system response is included in Section 5. Section 6 details the brief results of the present work and ends with concluding comments and future scope.

\section{Construction of three spring model}

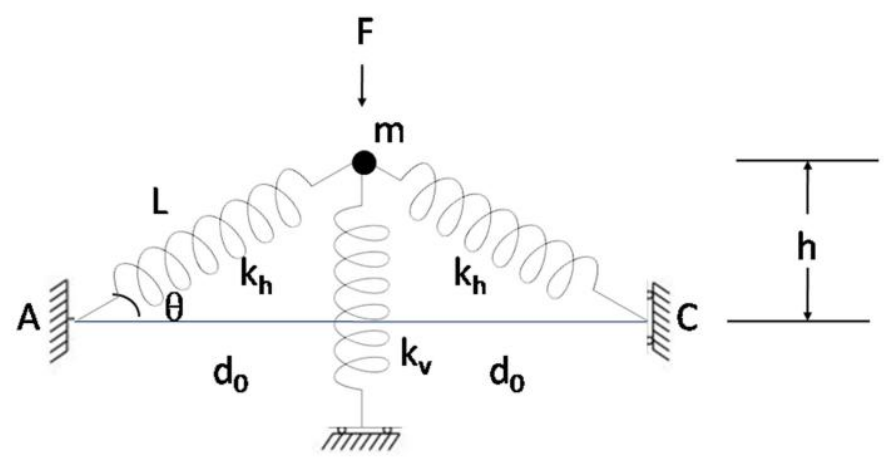

Figure 1: Three-spring system arrangement

Figure 1 shows a simple model of a nonlinear three spring system with two identical linear elastic springs each of length $L_{0}$ having equal stiffness $k_{h}$ are joined at one end say B to hold the mass and other two ends are separately hinged at two different supports A and C. To understand the forced vibration of the three-spring system under QZS condition shown in Fig.(1), we make an attempt to construct the system by a particular set of geometric parameters say $d_{0}=0.3 m, L=0.5 m$ which corresponds to the inclined angle $\theta_{0}=53^{0}$ [9].

When in operation, the system includes viscous damper with damping coefficient $\xi$ in parallel with the vertical spring which supports a mass $m$ is kept initially in the static equilibrium position. To emphasize the physical behavior of the system it is subjected to the harmonic excitation. The equation of motion for the system carrying Cubic Quintic septic nonlinear forces undergoes an excitation force $f_{e}$ is given by [9]

$$
\ddot{x}(t)+\zeta \dot{x}(t)+\alpha x(t)-\beta x^{3}(t)+\gamma x^{5}(t)-\delta x^{7}(t)=f_{e}=F \cos \Omega t,
$$

where $\zeta$ is the damping coefficient, $\alpha=\frac{k}{m}$ is the linear coefficient and $\beta=\frac{k}{m d_{0}^{2}}, \gamma=\frac{3}{4} \frac{k}{m d_{0}^{4}}$,

$\delta=\frac{15}{24} \frac{k}{m d_{0}^{6}}$ are the cubic, quintic, septic power coefficients respectively.

Our interest is to find the real system dynamics about the static equilibrium position by assigning a set of values for the geometric parameters $k=200 \mathrm{~N} / \mathrm{m}, d_{0}=0.3 \mathrm{~m}, \mathrm{~m}=$ $2.8 \mathrm{~kg}, L=0.5 \mathrm{~m}$. To examine this we substitute the values of $k, d_{0}, m, L$ in $\alpha, \beta, \gamma, \delta$ to obtain

$$
\ddot{x}+\zeta \dot{x}(t)+71.43 x-793.65 x^{3}+6613.75 x^{5}-30619.24 x^{7}=F \cos \Omega t .
$$


In Eq. (2) one expects a large amplitude of system oscillations for large nonlinear parameters. Applying the HAM to find approximate expressions for amplitude, phase shift and frequency-amplitude relationship. Moreover, free positively damped oscillatory systems are generally related to two different time scales. One for the frequency of oscillation and other to the decaying amplitude of oscillation. Thus, we define the two time scales as $\tau_{1}=\omega t$, $\tau_{2}=\epsilon t$, where $\omega$ and $\epsilon$ are unknown time constants. We can write Equation 1 explicity as below under the above transformation

$$
\begin{aligned}
& \omega^{2} \frac{\partial^{2} x(t)}{\partial \tau_{1}^{2}}+2 \omega \epsilon \frac{\partial^{2} x(t)}{\partial \tau_{1} \partial \tau_{2}}+\epsilon^{2} \frac{\partial^{2} x(t)}{\partial \tau_{2}^{2}}+\zeta\left(\frac{\partial x(t)}{\partial \tau_{1}}+\epsilon \frac{\partial x(t)}{\partial \tau_{2}}\right) \\
& +\alpha x(t)-\beta x^{3}(t)+\gamma x^{5}(t)-\delta x^{7}(t)=F \cos \left(\tau_{1}+\tau_{2}\right)
\end{aligned}
$$

subject to initial conditions

when $\tau_{1}=\tau_{2}=0$.

$$
x\left(\tau_{1}, \tau_{2}\right)=a, \dot{x}\left(\tau_{1}, \tau_{2}\right)=0
$$

\section{Application of Homotopy Analysis Method}

In this section we aim to derive the analytical solution for the nonlinear frequency response of the forced vibration in a typical three spring oscillator governed by Eq. (1). The procedure for the HAM is defined in detail before by Liao, starts with the "Rule of solution expression" which means a HAM series solution can be represented by a power series. It is based on the continuous variation from the initial guess to the exact solution of the considered [8]. Here we have chosen the initial guess to the solution of Eq. (1) of the following form:

$$
x\left(\tau_{1}, \tau_{2}\right)=A\left(\tau_{2}\right) \exp \left(i \tau_{1}\right)+A^{*}\left(\tau_{2}\right) \exp \left(i \tau_{1}\right),
$$

where $A\left(\tau_{2}\right)$ and $A^{*}\left(\tau_{2}\right)$ are the complex conjugate constants. For the considered problem, one introduces an embedding parameter $q$ and construct a transformation in such a way that $x\left(\tau_{1}, \tau_{2}, q\right)$ continuously varies from the initial guess $x_{0}\left(\tau_{1}, \tau_{2}\right)$ to their exact solution $x\left(\tau_{1}, \tau_{2}\right)$ as $q$ increases from 0 to 1 . In line with this reason, we can replace $x\left(\tau_{1}, \tau_{2}\right)$ in Equation 3 with the variation $x\left(\tau_{1}, \tau_{2}, q\right)$ in order to construct a linear and nonlinear differential operators in $q$.

The auxiliary linear operator is given by

and the nonlinear operator

$$
L\left[x_{0}\left(\tau_{1}, \tau_{2}, q\right)\right]=\omega_{0}^{2} \frac{\partial^{2} x}{\partial \tau_{1}^{2}}+2 \omega_{0} \epsilon_{0} \frac{\partial^{2} x}{\partial \tau_{1} \partial \tau_{2}}+\epsilon_{0}^{2} \frac{\partial^{2} x}{\partial \tau_{2}^{2}}+\alpha x_{0}
$$

$$
\begin{aligned}
& N\left[x\left(\tau_{1}, \tau_{2}, q\right)\right]=\Omega_{1}^{2} \frac{\partial^{2} x}{\partial \tau_{1}^{2}}+2 \Omega_{1} \Lambda_{1} \frac{\partial^{2} x}{\partial \tau_{1} \partial \tau_{2}}+\Lambda_{1}^{2} \frac{\partial^{2} x}{\partial \tau_{2}^{2}}+\alpha x_{0} \\
& +\zeta\left(\Omega_{1} \frac{\partial x}{\partial \tau_{1}}+\Lambda_{1} \frac{\partial x}{\partial \tau_{2}}\right)+\alpha x-\beta x^{3}+\gamma x^{5}-\delta x^{7}-F \cos \left(\tau_{1}+\tau_{2}\right) .
\end{aligned}
$$

The procedure of this method continues with the definition of the zeroth order deformation equation described as below:

$$
(1-q) L\left[x\left(\tau_{1}, \tau_{2}, q\right)-x_{0}\left(\tau_{1}, \tau_{2}\right)\right]=p N\left[x\left(\tau_{1}, \tau_{2}, q\right)\right]
$$

where $p$ is known as the convergence control parameter such that a properly chosen $p$ converges the series at $q=1$. When $q=0$, Eq. (8) has the solution with

$$
\Omega_{1}(0)=\omega_{0}, \Lambda_{1}(0)=\epsilon_{0} .
$$

With the nature of Eq. (9) one can write $x\left(\tau_{1}, \tau_{2}\right)$ as

$$
x\left(\tau_{1}, \tau_{2}, q\right)=\left.\Sigma_{k=0}^{\infty} \frac{1}{k !} \frac{\partial^{k} \omega(q)}{\partial q^{k}}\right|_{q=0} q^{k} .
$$


The $k^{t h}$ order deformation equation from Eq.(8) takes the form

$$
\begin{aligned}
& L\left[x_{k}\left(\tau_{1}, \tau_{2}\right)-\chi_{k} x_{k-1}\left(\tau_{1}, \tau_{2}\right)\right]=p R_{k}\left(\tau_{1}, \tau_{2}\right)-M_{k}\left(\tau_{1}, \tau_{2}\right)+ \\
& \chi_{k-1}\left(\tau_{1}, \tau_{2}\right)+M_{k-1}\left(\tau_{1}, \tau_{2}\right),
\end{aligned}
$$

where

$$
\begin{aligned}
& M_{k}\left(\tau_{1}, \tau_{2}\right)=\Sigma_{m=1}^{k}\left[\left(\Sigma_{j=0}^{k} \omega_{j} \omega_{m-j}\right) \frac{\partial^{2} x_{k-m}}{\partial \tau_{1}^{2}}+2\left(\Sigma_{j=0}^{m} \omega_{j} \epsilon_{m-j}\right) \frac{\partial^{2} x_{k-m}}{\partial \tau_{1} \partial \tau_{2}}\right. \\
& \left.+\left(\sum_{j=0}^{m} \epsilon_{j} \epsilon_{m-j}\right) \frac{\partial^{2} x_{k-m}}{\partial \tau_{2}^{2}}+\zeta\left(\omega_{m} \frac{\partial x_{k-m}}{\partial \tau_{1}}+\epsilon_{m} \frac{\partial x_{k-m}}{\partial \tau_{2}}\right)\right], \\
& R_{k}\left(\tau_{1}, \tau_{2}\right)=\left.\frac{1}{(k-1) !} \frac{d^{k-1} N(x)}{d q^{k-1}}\right|_{q=0}
\end{aligned}
$$

with $\chi_{k}=0$ for $k \leq 1$ and $\chi_{k}=1$ for $k>1$. In an iterative basis one can solve the first order deformation equation for $\tau_{1}, \tau_{2}$ by setting $k=1$. Eq. (9) takes the form

and

$$
L\left[x_{k}\left(\tau_{1}, \tau_{2}\right)-x_{0}\left(\tau_{1}, \tau_{2}\right)\right]=p R_{1}\left(\tau_{1}, \tau_{2}\right)-M_{1}\left(\tau_{1}, \tau_{2}\right)
$$

$$
\begin{aligned}
& R_{1}=\omega_{0}^{2} \frac{\partial^{2} x_{0}}{\partial \tau_{1}^{2}}+2 \omega_{0} \epsilon_{0} \frac{\partial^{2} x_{0}}{\partial \tau_{1} \partial \tau_{2}}+\epsilon_{0}^{2} \frac{\partial^{2} x_{0}}{\partial \tau_{2}^{2}}+\alpha x_{0}-\beta x_{0}^{3}+\gamma x_{0}^{5}-\delta x_{0}^{7} \\
& -F \cos \left(\tau_{1}+\sigma \tau_{2}\right), \\
& M_{1}=2 \omega_{0} \omega_{1} \frac{\partial^{2} x_{0}}{\partial \tau_{1}^{2}}+2\left(\omega_{0} \epsilon_{1}+\omega_{1} \epsilon_{0}\right) \frac{\partial^{2} x_{0}}{\partial \tau_{1} \partial \tau_{2}}+2 \epsilon_{0} \epsilon_{1} \frac{\partial^{2} x_{0}}{\partial \tau_{2}^{2}}+\omega_{0} \omega_{1} x_{0} .
\end{aligned}
$$

In order to have a bounded solution, the coefficients of secular terms in the right hade of Eq. (16) should be assigned to zero. This results in two linear algebraic equations in $A$ and $A^{*}$. On assigning $A=\frac{1}{2} a\left[\tau_{2}\right] \exp \left(i\left(\phi\left[\tau_{2}\right]+\epsilon \sigma\right)\right)$ where $\phi$ is the phase shift of the response and solve them to find the unknown $a$ which is the real amplitude of the response. After eliminating secular and constant terms we arrive at the expression shown below:

$$
\begin{aligned}
& F^{2}=\left(\frac{1}{2} p \alpha-\frac{3}{8} p \beta a^{3}+\frac{5}{16} p \gamma a^{5}-\frac{35}{128} p \delta a^{7}-\frac{1}{2} p \sigma^{2} \epsilon_{0}^{2} a\right. \\
& +\sigma^{2} \epsilon_{0} \epsilon_{1} a-\sigma p \epsilon_{0} \omega_{0} a+\sigma \epsilon_{1} \omega_{0} a-\frac{1}{2} p \omega_{0}^{2} a+\sigma \epsilon_{0} \omega_{1} a \\
& \left.+\frac{1}{2} \omega_{1} a\right)^{2}+\left(-\frac{1}{2} p \epsilon_{0} \sigma \zeta a-\frac{1}{2} p \zeta \omega_{0} a\right)^{2}
\end{aligned}
$$

Rewriting it in terms of $\Omega=\omega_{0}+\epsilon \sigma$

$$
\frac{\Omega}{\omega_{0}}=1+\frac{3 \epsilon \beta a^{2}}{8 \omega_{0}^{2}}+\frac{5 \epsilon \gamma a^{4}}{16 \omega_{0}^{2}}+\frac{35 \epsilon \delta a^{6}}{128 \omega_{0}^{2}} \pm \sqrt{\frac{F \epsilon^{2}}{4 a^{2} \omega_{0}^{4}}-\frac{\epsilon^{2} \zeta^{2}}{4 \omega_{0}^{2}}-\frac{B}{2\left(\frac{1}{16 \epsilon^{2}}\right)}},
$$

where

$$
B=-\left(\frac{1}{8 \epsilon^{2}}+\frac{1}{16 \epsilon}\left(\frac{P+Q}{\omega_{0}}\right)\right) \pm \sqrt{\left(\frac{1}{8 \epsilon^{2}}+\frac{1}{16 \epsilon}\left(\frac{P+Q}{\omega_{0}}\right)\right)^{2}-4\left(\frac{1}{16 \epsilon^{2}}\right)\left[\left(\frac{1}{\epsilon^{2}}\right)-\frac{P+Q}{\epsilon \omega_{0}}+\frac{P Q}{\omega_{0}^{2}}+\frac{\zeta^{2} \omega_{0}}{4}\right]},
$$

$P=\frac{3 \beta a^{2}}{8 \omega_{0}}+\frac{5 \gamma a^{4}}{16 \omega_{0}}+\frac{35 \delta a^{6}}{128 \omega_{0}}, \quad Q=\frac{-9 \beta a^{2}}{8 \omega_{0}}-\frac{25 \gamma a^{4}}{16 \omega_{0}}-\frac{245 \delta a^{6}}{128 \omega_{0}}$ and $\sigma$ is the small detuning parameter; $\epsilon$ and $\omega$ are the decaying amplitude and loading frequency of harmonic oscillation close to the natural frequency of the system respectively. The Eq. (17) is regarded as the analytical frequency response of Cubic Quintic Septic Duffing oscillator obtained by the first order HAM approach. 


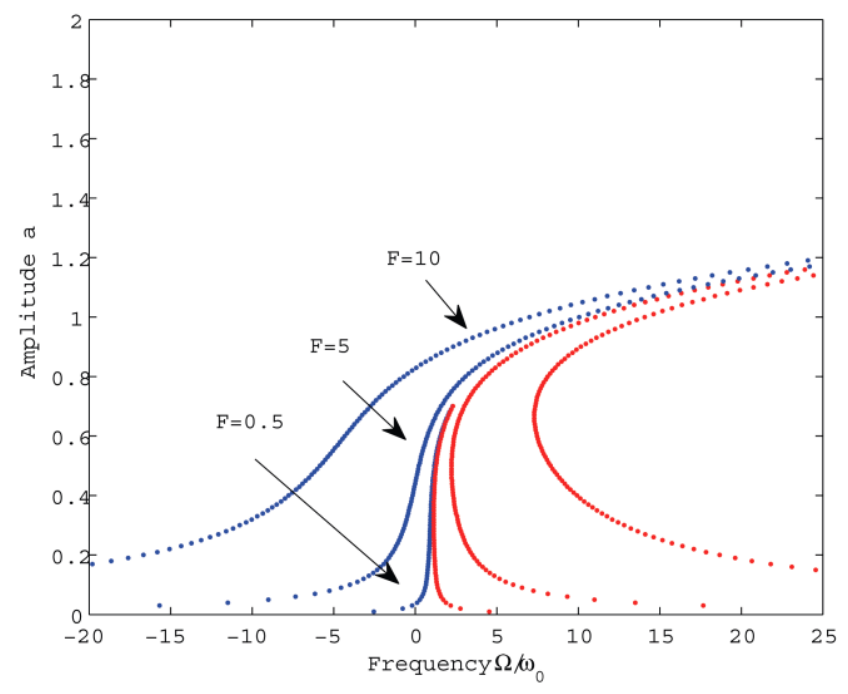

Figure 2: The magnification factor of Cubic Quintic Septic Duffing Oscillator with driving force $F=0.5, F=5, F=10, \zeta=0.5$ for Parameter values $\alpha=71.43, \beta=-793.65, \gamma=$ $6613.75, \delta=-30619.24$

\section{Frequency Domain behaviour}

Solving Eq. (17) for $a$ at different values of $\omega_{0}$ gives the amplitude response of the system in the frequency domain. The primary resonance of the system is studied under forcing function as the control parameter is shown in Fig. (2). The system exhibits hysteresis due to multiple solution for different damping $\zeta$ and forcing $F$ parameters, and in practice never be determined experimentally. As the excitation amplitude increases as $F=0.5, F=5, F=10$ for constant damping ratio $\zeta=0.05$ the corresponding peak amplitude of the response curve also increases and lean more towards the higher frequency region.

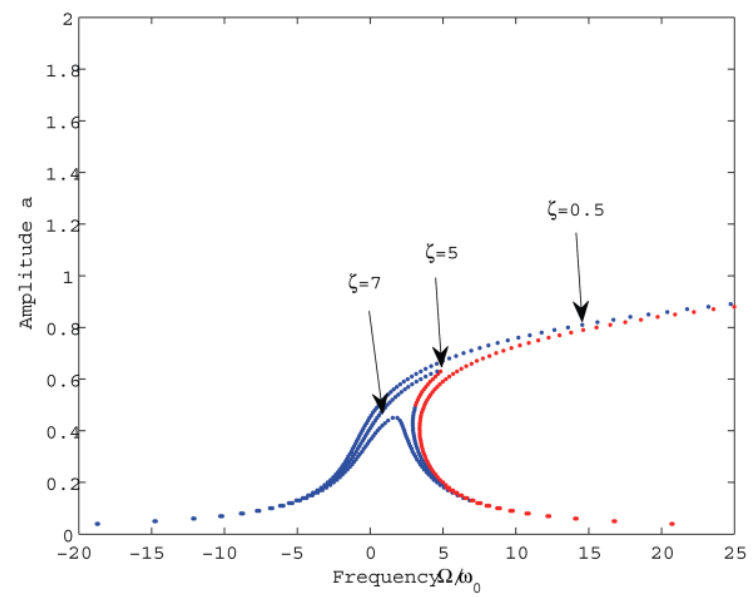

Figure 3: Variation of the amplitude response with respect to the excitation frequency for different values of the damping ratio $\zeta=0.5, \zeta=5, \zeta=7$ for the parameters $\alpha=$ $71.43, \beta=-793.65, \gamma=6613.75, \delta=-30619.24$

This shows the hardening characteristic of the spring because of the overall positive value of the nonlinear Cubic, Quintic and Septic terms in the governing equation of the oscillator. Also, Fig. (3) shows the change in the steady-state amplitude of the system for the given excitation frequency $F=10$. The influence of damping and driving frequency on the 
magnification factor conveys the strength of nonlinearity in the amplitude response of the governing equation. This multivalue response of the Duffing oscillator is as a consequence of the nonlinear Septic term in Eq. (1). In Fig. (4) the amplitude response behaviour of the Cubic Quintic Septic Duffing oscillator compare with Cubic Quintic and Cubic oscillators are shown. Unlike the amplitude response in the Cubic Quintic and Cubic case, the amplitude response in the Cubic Quintic Septic case is more nonlinear. The more positive the nonlinearity, the higher is the shift of the peak value of the magnification factor away from $\Omega=1$ towards higher frequencies.

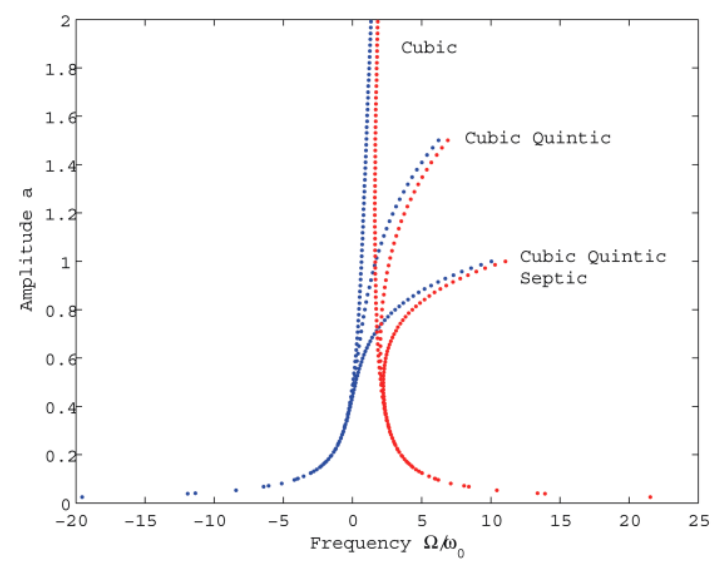

Figure 4: Comparison of Cubic Quintic Septic Duffing oscillator with Cubic Quintic and Cubic oscillators

The transient solution of the forced Duffing oscillator whose amplitude decays exponentially in time that depends on the initial conditions is given in Fig. 5(a). The corresponding phase plot in Fig. 5(b) for specific initial condition depicts the system is nonlinear and unstable, and the frequency response curve for $F=5$ with $\zeta=0.05$ illustrates jump phenomenon which means the amplitude response has a discontinuity at $\Omega=1.4$ is plotted in Fig. 5(c). One cannot observe any solution in the instability region which is denoted as red dots due to ranges of solutions for each $\Omega$, when the system is perturbed. This results subcritical bifurcation in the frequency domain at $\Omega=1.4$.
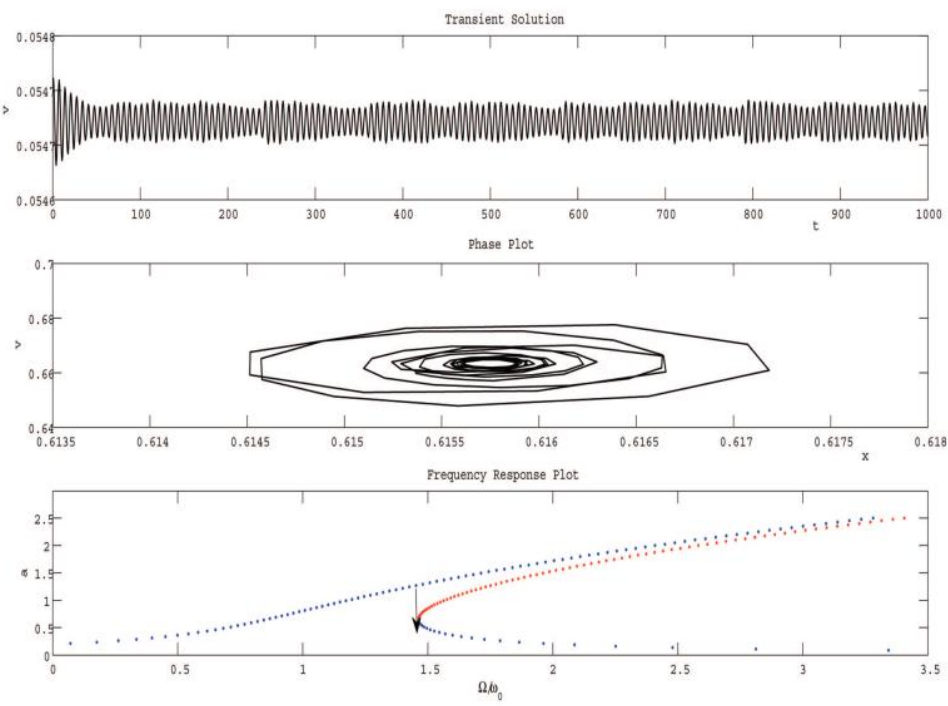

Figure 5: a) Transient solution, b) Phase plot, c) Frequency response of Cubic Quintic Septic Duffing oscillator

5 Time domain behaviour 
As seen in the frequency domain, the Duffing equation also exhibits interesting phenomena in the time domain as the control parameter varies. The time curve and Phase portraits offer an effective view of the dynamics of the system at various initial conditions in this parameter space. Using $F$ as the control parameter, with $\zeta=1, \alpha=71.43, \beta=$ $-793.65, \gamma=6613.75, \delta=-30619.24$ and $\Omega=1$, the solution transitions from having one stable limit cycle to half stable and unstable limit cycles for different initial conditions is detailed in Fig. (6). In the case of Fig. 6(a) all the neighboring trajectories for $F=0.01$ with different initial conditions approach the limit cycle as time approaches infinity. This is a stable limit cycle where the system imply self sustained oscillations. There is also a semi stable limit cycle observed for $F=0.34$ in which, the trajectories from outside stick to limit cycle whereas the inside trajectories would be the family of other cycles is represented in Fig. 7. The Fig. 8 represents the unstable limit cycle where the trajectories may approaches the limit cycle at negative infinity. Generally, only the nonlinear systems exhibit the property of limit cycle behavior. The corresponding time series behavior for each $F$ with different initial conditions are also analysed.
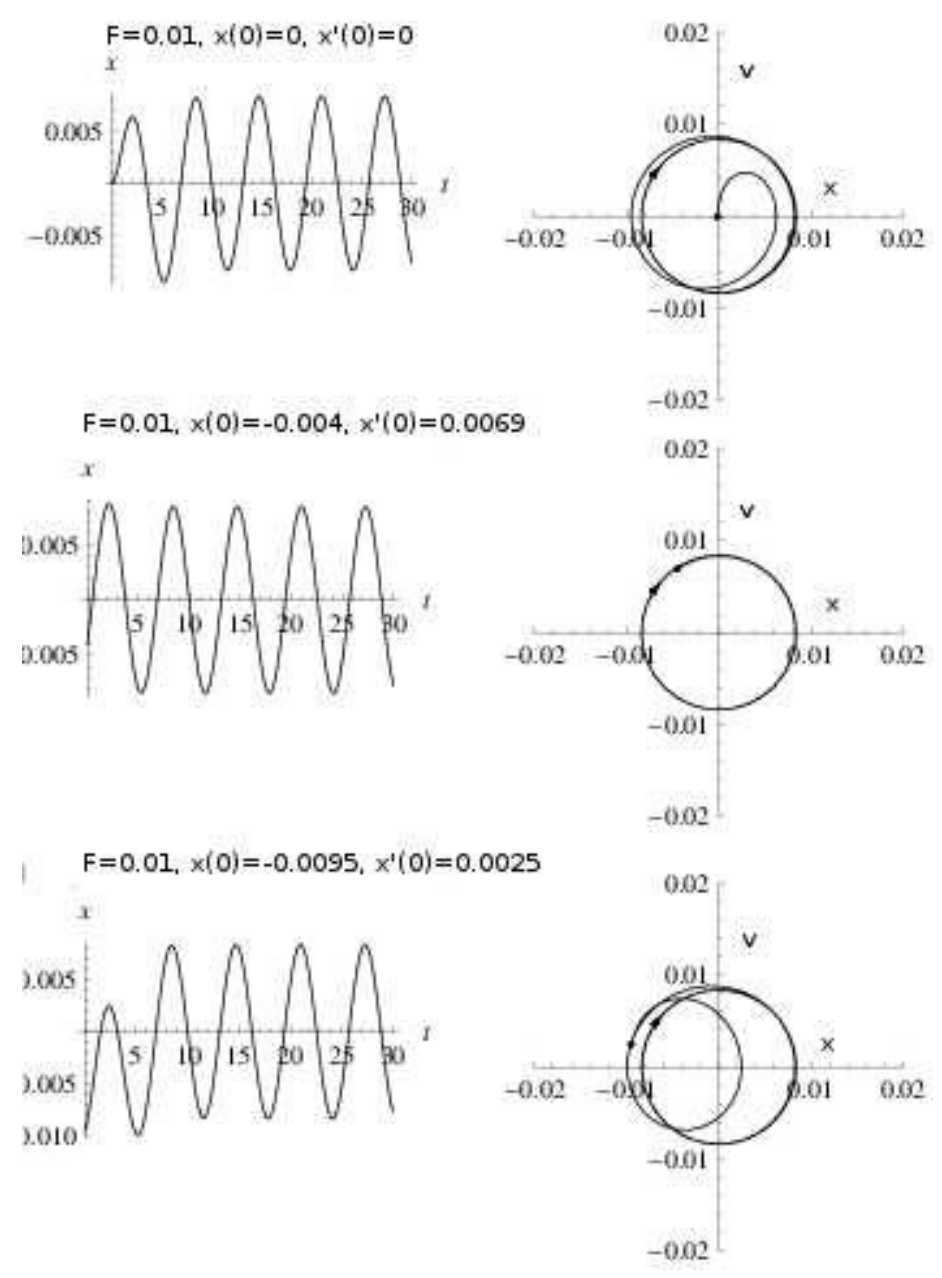

Figure 6: Time plot and phase plot for $F=0.01$ represent stable limit cycle 

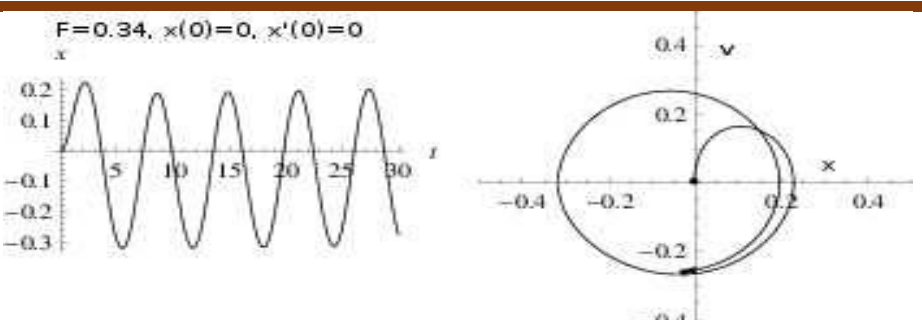

$F=0.34, x(0)=-0.1925, x^{\prime}(0)=0.2025$
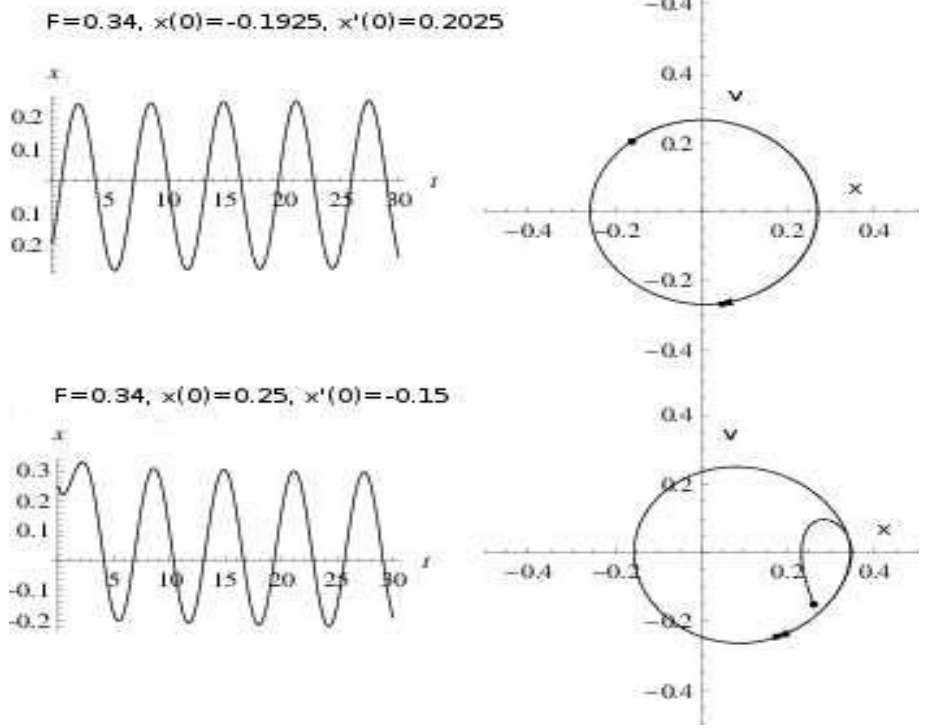

Figure 7: Time plot and phase plot for $F=0.34$ represent half stable limit cycle
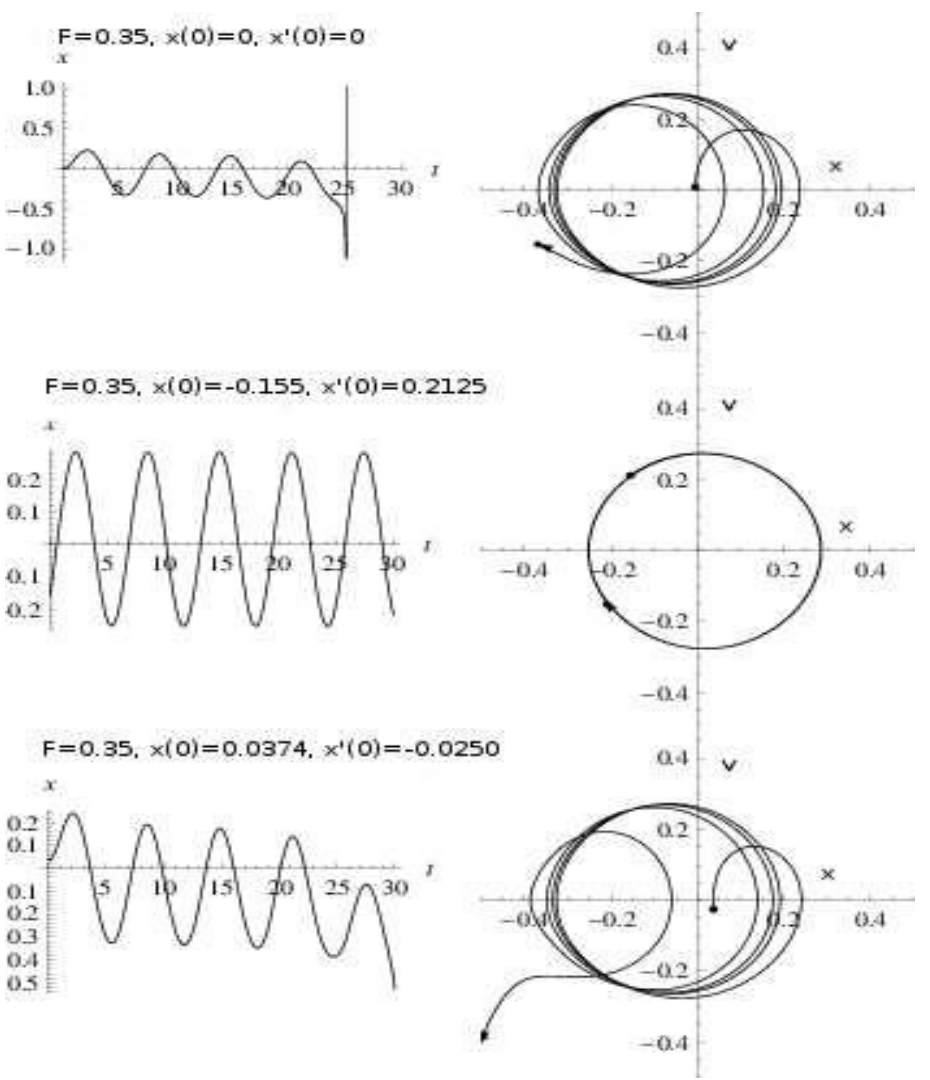

Figure 8: Time plot and phase plot for $F=0.35$ represent unstable limit cycle 


\section{Conclusion}

In the present work, a nonlinear model of three springs is considered with the geometrical parameters $d_{0}=0.3, L_{0}=0.5, \theta_{0}=53^{0}$ satisfying the QZS property at the equilibrium point. The system is forced at the static equilibrium position and the equation of motion for such system leads to a strong nonlinear non conservative Cubic Quintic Septic Duffing equation consisting of large nonlinear restoring force terms. The Homotopy Analyisis Method (HAM) is used to solve the equation for studing frequency response characteristics. This approach enables us to estabilish approximate expression for amplitude - frequency relation for the governing equation. The primary resonance of the system is studied using forcing function as the control parameter. The system exhibit jump phenomenon and the curve bends towards high frequency region which shows the hardening nature of the spring. Also, the system response is analysed for various damping ratio with respect to a particular excitation frequency and is found that the amplitude of the response decreases as damping increases. The comparison studies of the response of the Cubic Quintic Septic equation with the Cubic Quintic and Cubic Duffing oscillators affirm the strength of nonlinearity of the governing equation. Moreover, in time domain, the response possess limit cycles for various initial conditions. Also for increasing driving frequency the system trajectory changes from stable limit cycle to unstable limit cycle.

\section{References}

[1] Y. Ueda, In New Approaches to Nonlinear Problems in Dynamics (Editor P. J. Holmes): Pennsylvania: SIAM. Steady motions exhibited by Duffing's equation: a picture book of regular and chaotic motions, 1980

[2] M. N. Hamdan and T. D. Burton, On the steady state response and stability of nonlinear oscillators using harmonic balance, Journal of Sound and Vibration 166, 255 - 266, 1993

[3] W. Szemplinska-stupnicka, Bifurcation of harmonic solutions leading to chaotic motion in the softening type Duffing's oscillator, International Journal of Nonlinear Mechanics 53, 257-277, 1988

[4] S.J. Liao, The proposed homotopy analysis technique for the solution of nonlinear problems, PhD thesis, Shanghai Jiao Tong University, 1992.

[5] S.J. Liao, An explicit, totally analytic approximation of Blasius viscous flow problems, Int. J. Non-Linear Mech. 34 (4),759-778, 1999

[6] S.J. Liao, A simple way to enlarge the convergence region of perturbation approximations, Int. J . Non-linear Dynam. 19 (2), 93-110, 1999

[7] S.J. Liao, A uniformly valid analytic solution of 2D viscous flow past a semi-infinite flat plate, J. Fluid Mech. 385, 101-128, 1999

[8] S.J. Liao, On the homotopy analysis method for nonlinear problems, Applied Mathematics and Computation 147 499-513, 2004

[9] S. K. Remmi, M. M. Latha, Cubic Quintic Septic Duffing Oscillator: An analytical study, Chinese Journal of Physics, DOI: 10.1016/j.cjph.2018.08.009. 